\title{
Multifrequency observations of KAZ 102 during the ROSAT all-sky survey
}

\section{Citation}

Treves, A., H. H. Fink, M. Malkan, S. J. Wagner, B. J. Wilkes, F. Baganoff, J. Heidt, et al. 1995. "Multifrequency Observations of KAZ 102 During the ROSAT All-Sky Survey." The Astrophysical Journal 442 (April): 589. doi:10.1086/175462.

\section{Published Version}

doi:10.1086/175462

\section{Permanent link}

http://nrs.harvard.edu/urn-3:HUL.InstRepos:30212184

\section{Terms of Use}

This article was downloaded from Harvard University's DASH repository, and is made available under the terms and conditions applicable to Other Posted Material, as set forth at http:// nrs.harvard.edu/urn-3:HUL.InstRepos:dash.current.terms-of-use\#LAA

\section{Share Your Story}

The Harvard community has made this article openly available.

Please share how this access benefits you. Submit a story.

Accessibility 


\title{
MULTIFREQUENCY OBSERVATIONS OF KAZ 102 DURING THE ROSAT ALL-SKY SURVEY
}

\author{
A. Treves, ${ }^{1}$ H. H. Fink, ${ }^{2}$ M. Malkan, ${ }^{3}$ S. J. Wagner,${ }^{4}$ B. J. Wilkes, ${ }^{5}$ F. Baganoff, ${ }^{3}$ J. Heidt, ${ }^{4}$ E. Pian, ${ }^{1}$ \\ A. Sadun, ${ }^{6}$ S. Schaeidt, ${ }^{2}$ J. T. Bonnell, ${ }^{7}$ W. Brinkmann, ${ }^{2}$ D. De Martino, ${ }^{8}$ L. Maraschi, ${ }^{9}$ \\ E. G. TANZI, ${ }^{10}$ M. H. UlRICH, ${ }^{11}$ AND W. WAMSTEKER ${ }^{8}$ \\ Received 1994 June 13; accepted 1994 October 10
}

\begin{abstract}
The bright quasar Kaz 102, which lies in the vicinity of the North Ecliptic Pole, was monitored during the ROSAT All Sky Survey for 121.5 days from 1990 July 30 to 1991 January 25. In the course of the survey, optical photometry with various filters was performed at several epochs, together with UV (IUE) and optical spectrophotometry. The spectral energy distribution in the $3 \times 10^{14}-3 \times 10^{17} \mathrm{~Hz}$ range is obtained simultaneously among the various frequencies to $\lesssim 1$ day. No clear case of variability can be made in the X-rays, while in the optical and UV variability of $10 \%-20 \%$ is apparent. An analysis of IUE and Einstein archives indicates a doubling timescale of years for the UV and soft X-ray flux. The X-ray photon index, which in 1979 was rather flat $\left(\Gamma=0.8_{-0.4}^{+0.6}\right)$, in $1990 / 1991$ was found to be $\Gamma=2.22 \pm 0.13$, a typical value for radio-quiet quasars in this energy range. The overall energy distribution and the variability are discussed.
\end{abstract}

Subject headings: galaxies: active - quasars: individual ( Kaz 102) - ultraviolet: galaxies — X-rays: galaxies

\section{INTRODUCTION}

During the six month survey phase ROSAT observed the north and south ecliptic poles once per orbit with the PSPC experiment. The regions in the vicinity of the poles were therefore monitored for a duration unprecedented for X-ray telescopes. Because of this unique opportunity, multifrequency observations of active galactic nuclei in the polar region were organized, with the aim of studying correlated variability (see, e.g. Brinkmann \& Trümper 1992).

In this paper, we examine the close-by, radio-quiet quasar $\mathrm{Kaz} 102\left(m_{B} \simeq 16, z=0.136\right)$, which is a strong $\mathrm{X}$-ray source and has the hardest spectrum of any quasar observed with the Einstein Observatory (Wilkes \& Elvis 1987). Preliminary results were reported by Treves et al. (1992), and by Walter \& Fink (1993).

The paper is structured as follows: in $\S 2$ the $R O S A T$ X-ray light curve and spectrum are presented and compared with Einstein observations. Optical light curves and optical and UV spectrophotometry are described in $\S 3$. The results are discussed in $\S 4$.

\footnotetext{
${ }^{1}$ Scuola Internazionale Superiore di Studi Avanzati, Via Beirut 2-4, 34014 Trieste, Italy.

${ }^{2}$ Max Planck-Institute für Extraterrestriche Physik, Giessenbachstrasse, 85740 Garching bei München, Germany.

${ }^{3}$ University of California, Los Angeles, CA 90024-1562.

${ }^{4}$ Landessternwarte Heidelberg-Königstuhl, Königstuhl, 69117 Heidelberg, Germany.

${ }_{5}^{5}$ Harvard-Smithsonian Center for Astrophysics, 60 Garden Street, Cambridge, MA 02138

${ }^{6}$ Department of Physics and Astronomy, Agnes Scott College, 141 East College Avenue, Decatur, GA 30030.

7 Goddard Space Flight Center, Greenbelt, MD 20771.

${ }^{8}$ ESA, IUE Observatory, P.O. Box 50727, 28080 Madrid, Spain.

9 Dip. di Fisica, Università de Genova, Via Dodecaneso 33, 16133 Genova, Italy.

${ }^{10}$ Istituto di Fisica Cosmica, Consiglio Nazionale delle Ricerche, Via Bassini 15, 20133 Milano, Italy.

${ }^{11}$ European Southern Observatory, Karl-Schwarzschild-Strasse 2, 85748 Garching bei München, Germany.
}

\section{X-RAY OBSERVATIONS}

\subsection{Light Curve}

The source, which lies at $\sim 1^{\circ}$ from the north ecliptic pole, was observed by ROSAT during the survey phase for 121.5 days, in two parts: (1) from 1990 July 30 to October 31 and (2) from 1990 December 27 to 1991 January 25. Each exposure had a typical effective (i.e., on-axis) duration of 10-30 s, and the separation between the exposures was the satellite orbital period of 96 minutes. A special procedure was used for the extraction of survey data. The source + background counts were estimated from a $300^{\prime \prime}$ radius circle centered on the X-ray source position with the background taken from a $610^{\prime \prime}$ radius circle separated from the source by $17^{\prime}$. The net raw source + background counts were $\sim 6100$, with an estimated background contribution of 1600 counts. The effective exposure time was $\sim 24,000 \mathrm{~s}$. Taking into account vignetting corrections derived from energy dependent flat-field exposures of the detector's field of view (Snowden et al. 1994) this yields an average source count rate of $\sim 0.3$ counts $s^{-1}$ superposed on a background of $\sim 0.1$ counts $\mathrm{s}^{-1}$. While the source was marginally observable during each orbit, integrations over a day or more are required in order to reduce the statistical error. Figure $1 a$ gives the light curve with a two-day integration. Only statistical errors are reported. The reduced $\chi^{2}$ under the hypothesis of constancy is 1.3 , with a formal probability of constancy of $4.6 \%$. However, systematic study of light curves of a complete survey sample shows that variability should not be considered as reliable for $\chi^{2} \lesssim 2$ (Schaeidt 1994). Therefore we conclude that no solid case for variability can be made. We note the appearance of possible dips at MJD $=8125$ and at $\mathrm{MJD}=8160($ Figure $1 a)$.

\section{2. $X$-Ray Spectrum}

A standard minimization procedure was used to fit the background corrected spectrum, from the cumulative exposure, to the following models: (1) a single power-law model with absorption taken as a free parameter; and (2) a power law plus a blackbody model with absorption fixed at the Galactic value 


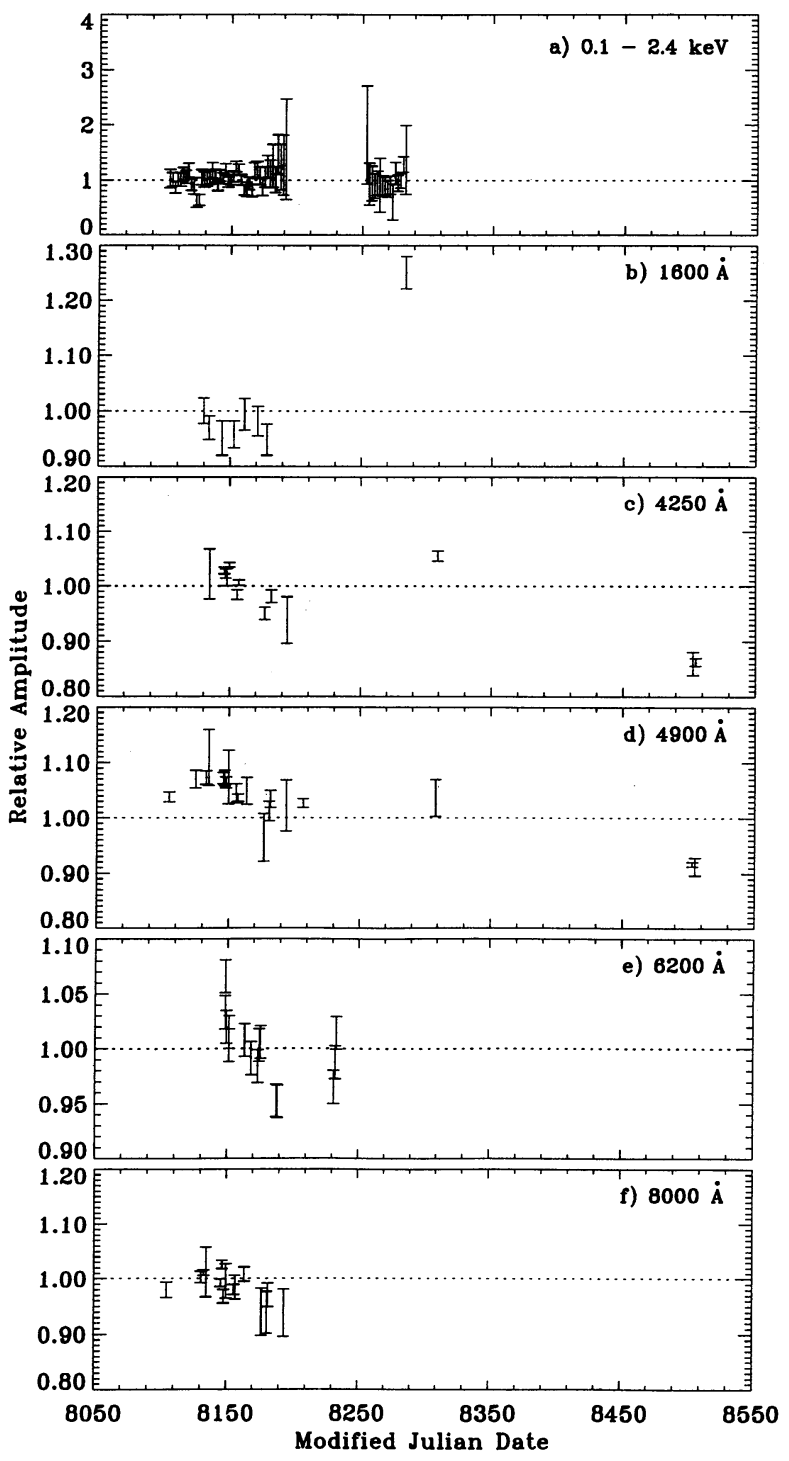

Fig. 1.- Light curves of Kaz 102 normalized to the average (see Table 5): (a) X-ray counts $\mathrm{s}^{-1}$; the data are binned with a 2 day flux integration; (b) UV; (c) $B$ band (from Lick observations); (d) $g$ band (from Lick observations); (e) $R$ band (from Heidelberg observations); $(f) I$ band (from Lick observations).

$\left[N_{\mathrm{H}}=(5.0 \pm 0.3) \times 10^{20} \mathrm{~cm}^{-2}\right.$, Elvis, Lockman, \& Wilkes 1989]. Details are given in Table 1 and Figure 2. The entire ROSAT sensitivity range $(0.1-2.4 \mathrm{keV})$ was considered. We verified that the power-law fit remains unchanged within the errors excluding the channels below $0.2 \mathrm{keV}$, which, in the opinion of some authors, may be affected by instrumental uncertainties. The results of the analysis are consistent with those reported by Treves et al. (1992) and Walter \& Fink (1993), which were based on the analysis of a fraction of the data examined here, using a preliminary form of the response matrices. Note that the hydrogen column density given by fit (a), $N_{\mathrm{H}}=(4.08 \pm 0.53) \times 10^{20} \mathrm{~cm}^{-2}$, is somewhat lower than the Galactic one.

The source was observed with the Einstein Observatory back in 1979 June and the spectrum was discussed in Wilkes \& Elvis (1987). Here we are presenting a new analysis of the spectrum based on somewhat improved calibration matrices. A total of $\sim 600$ source photons were detected. A best fit was searched for
TABLE 1

PARAMETERS OF THE ROSAT SPECTRUM ${ }^{\mathrm{a}}$

\begin{tabular}{|c|c|c|}
\hline Parameter & Power Law ${ }^{b}$ & Power Law + Blackbody ${ }^{c}$ \\
\hline $\begin{array}{l}A_{\mathrm{pl}_{\mathrm{f}}}^{\mathrm{d}} \ldots \ldots \ldots \ldots \ldots \ldots \\
A_{\mathrm{bb}} \ldots \ldots \ldots \ldots .\end{array}$ & $2.71 \pm 0.28^{e}$ & $\begin{array}{l}2.65 \\
0.58\end{array}$ \\
\hline$\Gamma \ldots \ldots \ldots \ldots$ & $2.22 \pm 0.13$ & $2.29 \pm 0.12$ \\
\hline $\begin{array}{l}N_{\mathrm{H}}^{\mathrm{g}} . \\
k T^{\mathrm{h}}\end{array}$ & $4.08 \pm 0.53$ & $5 . \overline{0}$ \\
\hline$\chi^{2} /$ d.o.f.............. & 0.879 & 0.843 \\
\hline
\end{tabular}

a The spectrum is deabsorbed after Morrison \& McCammon 1983.

${ }^{\mathrm{b}} f_{\mathrm{pl}}\left(E, A_{\mathrm{pl}}, \Gamma, E_{0}\right) d E=A_{\mathrm{pl}}\left(E / E_{0}\right)^{-\Gamma} d E ; E_{0}=2 \mathrm{keV}$.

c $f_{\mathrm{pl}}+f_{\mathrm{bb}}$, with $f_{\mathrm{bb}}\left(E, A_{\mathrm{bb}}, T\right) d E=\left[A_{\mathrm{bb}}(E / k T)^{2}\right] /\left[k T\left(e^{E / k T}\right.\right.$

$-1)] d E$.

d In $10^{-4}$ photons $\mathrm{s}^{-1} \mathrm{~cm}^{-2} \mathrm{keV}^{-1}$.

e The quoted errors represent $1 \sigma$ uncertainties on the fitted parameters.

${ }_{\mathrm{f}}^{\mathrm{I}}$ In photons $\mathrm{s}^{-1} \mathrm{~cm}^{-2}$

g Equivalent hydrogen column density in $10^{20} \mathrm{~cm}^{-2}$. For the $\mathrm{pl}+\mathrm{bb}$ fit, $N_{\mathrm{H}}$ is fixed at the Galactic value.

${ }^{\mathrm{h}}$ In electron volts.

with an absorbed power-law fit. We obtain $N_{\mathrm{H}}=\left(1.1_{-1.0}^{+7.8}\right)$ $\times 10^{20} \mathrm{~cm}^{-2}, \Gamma=0.8_{-0.4}^{+0.6}$ and $F_{1 \mathrm{kev}}=0.34 \mu \mathrm{Jy}\left(\chi^{2}=0.69\right)$. These parameters are consistent with the previous analysis of Wilkes \& Elvis (1987).

Comparing the Einstein flux with the average 1990/1991 flux from our ROSAT observations $\left(F_{1 \mathrm{kev}}=0.85 \mu \mathrm{Jy}\right)$, an increase at $1 \mathrm{keV}$ of more than a factor of 2 is apparent. The energy index $/ N_{\mathrm{H}}$ plots of the two observations, which are shown in Figure 3, indicate on the one hand the much reduced uncertainty of the ROSAT spectrum due to higher photon statistics and to superior resolution of the detector. On the other hand, a spectral variation of the source is strongly suggested, as from the fact that the plots are completely disjoint. As apparent from the figure, in the Einstein observation the column density is poorly constrained. Fixing its value at $N_{\mathrm{H}}=4.1 \times 10^{20}$ $\mathrm{cm}^{-2}$, the spectral indices for ROSAT and Einstein are, respectively, $\Gamma_{R}=2.20 \pm 0.06$ and $\Gamma_{E}=1.1 \pm 0.2(1 \sigma)$, yielding $\Delta \Gamma=1.10 \pm 0.21$. The variation in the spectral index therefore appears significant at the $5 \sigma$ level.

TABLE 2

OPTICAL AND UV SPECTROPHOTOMETRY

\begin{tabular}{|c|c|c|}
\hline Instrument & Date & Exposure Time $^{\mathrm{a}}$ \\
\hline \multicolumn{3}{|c|}{ Ground-based Observations } \\
\hline Lick & 1990 Aug 01 & 20 \\
\hline Lick & 1990 Aug 31 & 10 \\
\hline Lick & $1990 \mathrm{Sep} 15$ & 10 \\
\hline Lick & 1990 Sep 29 & 13 \\
\hline MMT & 1990 Oct 14 & 20 \\
\hline Lick ............. & $1991 \mathrm{Feb} 21$ & 10 \\
\hline \multicolumn{3}{|c|}{ IUE Observations } \\
\hline SWP 31032 . & 1987 May 23 & 395 \\
\hline LWP $10810 \ldots$ & 1987 May 22 & 385 \\
\hline SWP $39545 \ldots$ & 1990 Aug 27 & 435 \\
\hline SWP $39558 \ldots \ldots$ & 1990 Aug 31 & 388 \\
\hline SWP $39608 \ldots \ldots$ & 1990 Sep 10 & 428 \\
\hline SWP $39664 \ldots \ldots$ & 1990 Sep 19 & 420 \\
\hline SWP $39718 \ldots$. & 1990 Sep 27 & 423 \\
\hline SWP 39788 . & 1990 Oct 07 & 379 \\
\hline SWP $39831 \ldots \ldots$ & 1990 Oct 14 & 405 \\
\hline LWP $19011 \ldots \ldots$ & 1990 Oct 14 & 390 \\
\hline SWP $40961 \ldots \ldots$ & 1991 Jan 27 & 333 \\
\hline
\end{tabular}

${ }^{a}$ In minutes. 


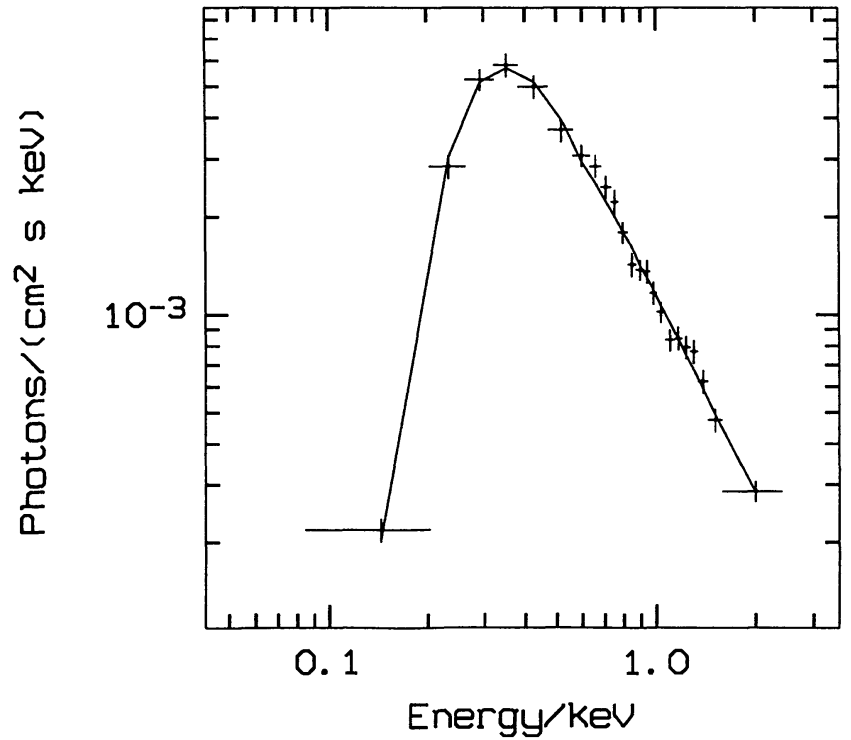

FIG. $2 a$

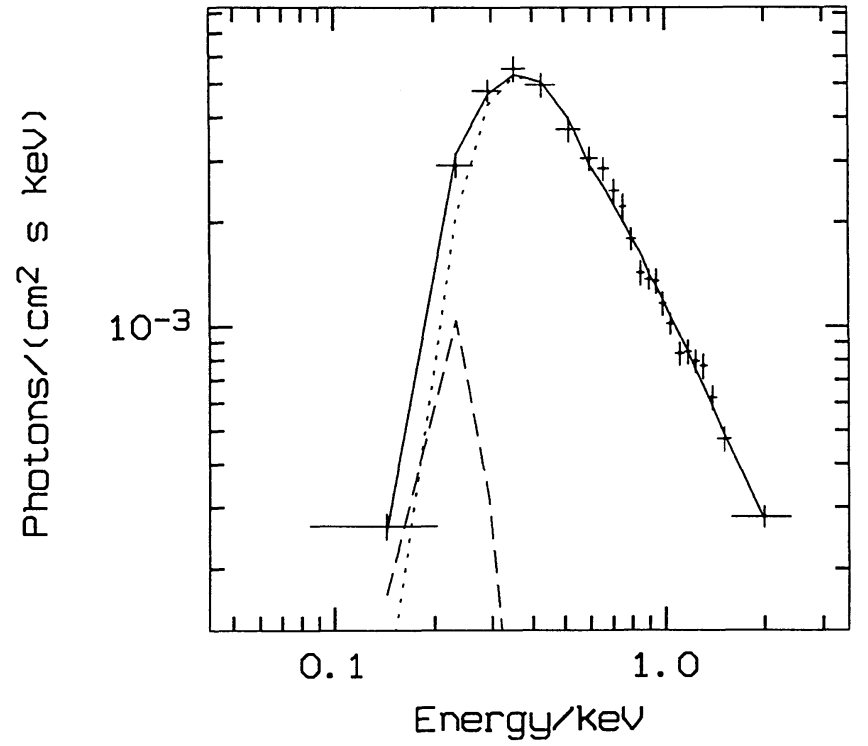

FIG. $2 b$

FIG. 2.-ROSAT average spectrum: (a) power-law fit plus absorption; $(b)$ power law plus blackbody fit with absorption fixed to the Galactic value. The dashed line represents the blackbody curve decomposed from the power law.

\section{OPTICAL-UV OBSERVATIONS}

\subsection{Spectroscopy}

Optical spectrophotometry of the source was performed at the Multiple Mirror Telescope (MMT) and at the Lick $3 \mathrm{~m}$ telescope (see Table 2, top section and Fig. 4).

The MMT spectrum was obtained using the blue spectrograph with a wavelength range $3200-6400 \AA$ and a resolution of $5 \AA\left(1^{\prime \prime} \times 3^{\prime \prime}\right.$ aperture, 300 grooves per millimeter grating).
Conditions were photometric and the spectrum was calibrated by taking a spectrum of both the quasar and a nearby flux standard star through a $5^{\prime \prime}$ diameter circular aperture. The small-aperture spectrum was then normalized to the continuum shape and level of the flux calibrated, large-aperture spectrum. We estimate the photometric errors to be $\sim 10 \%$.

The MMT spectrum can be directly compared with a spectrum of the source taken in April of 1988 with the Faint Object Grism Spectrograph (FOGS) on the MMT (Shastri et al. 1993).

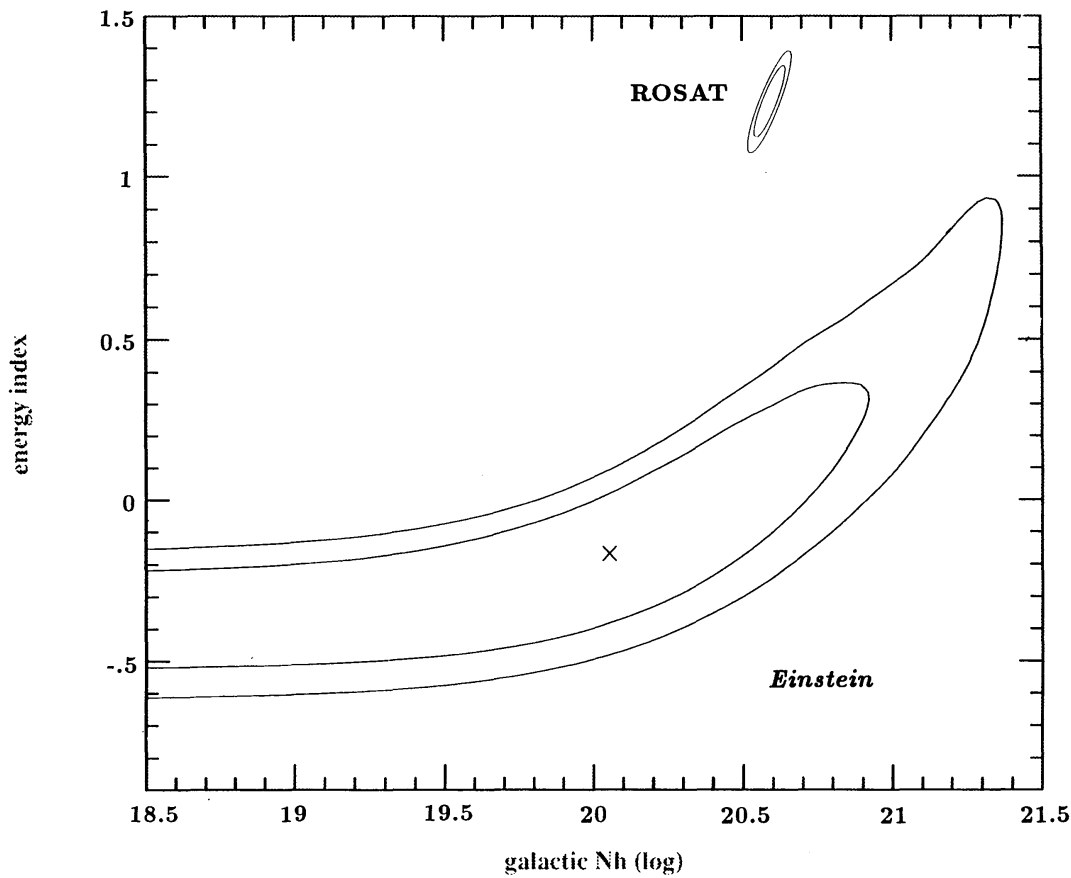

Fig. 3.-Energy index $/ N_{\mathrm{H}}$ fit contour of the Einstein spectrum compared with the one from ROSAT. The two curves of each contour represent the 1 and $1.5 \sigma$ confidence levels. 

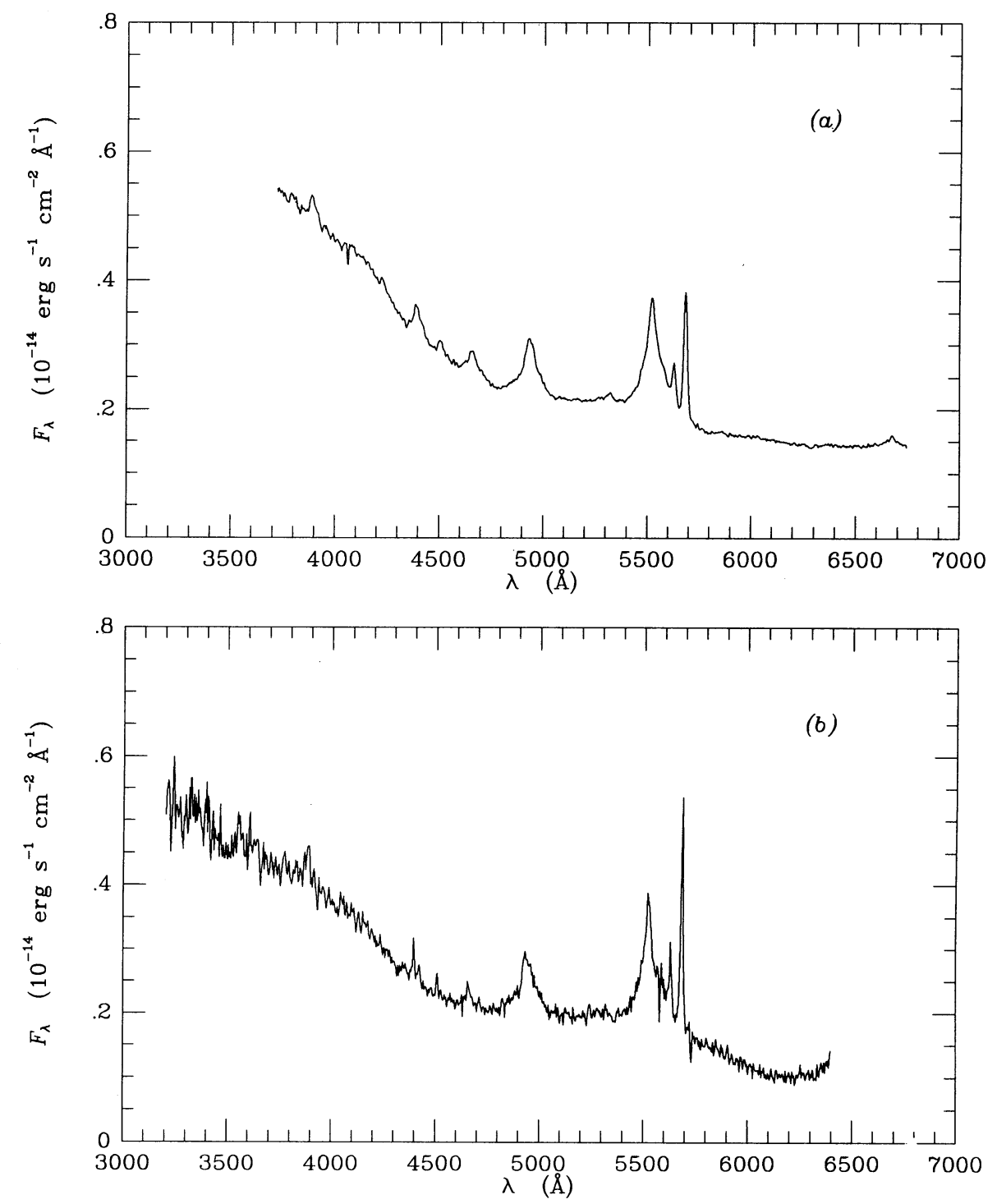

FIG. 4.-Optical spectrophotometry (see Table 2): (a) average of the five spectra from the Lick Observatory; (b) MMT spectrum

A comparison of the continuum level shows an increase of $\sim 10 \%$ in $2.5 \mathrm{yr}$. Given the estimated errors of $\sim 10 \%$, this change is not significant. Note that in Shastri et al. the ordinate scale of the spectrum should be corrected upward by a factor of 5 .

Five spectra of Kaz 102 were obtained with the Lick Observatory $3 \mathrm{~m}$ telescope using the UV/Schmidt Camera with a $5^{\prime \prime}$ slit. This instrument uses an $800 \times 800$ pixel TI CCD which is UV flooded before cool down for enhanced sensitivity at blue wavelengths. A $3001 \mathrm{~mm}^{-1}$ grating was used to cover the wavelength range from 3600 to $6600 \AA$ with a spectral resolution of $4 \AA$ pixel $^{-1}$. Standard star observations were made prior to and after observations of $\mathrm{Kaz} 102$. Between 3 and 7 spectrophotometric standard stars were observed during the course of each night. Good agreement between gain curves through out a night indicated that the August and September nights in 1990 were photometric. Only 1991 February 21 revealed the effects of variable cloud cover and seeing. For the August and September nights, the errors on spectrophotom- etry are estimated to be $5 \%-10 \%$.
The MMT spectrum is similar to the average one taken at the Lick Observatory, with an average discrepancy of $13.5 \%$ and a more marked percentage difference at shorter wavelengths, probably due to higher variability of the source at higher frequencies and to the nonsimultaneity of MMT and Lick spectrophotometry.

Nine $I U E$ spectra were gathered for this project with the

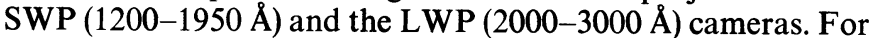
comparison, we have also retrieved two archival spectra from 1987 (see also Elvis et al. 1994). Details are given in Table 2, lower panel, and in Figure 5. The spectra were analyzed using an adaptation of the Gaussian Extraction Procedure (Urry \& Reichert 1988) to the MIDAS package and calibrated after Bohlin et al. (1990; SWP) and Cassatella, Lloyd, \& GonzalezRiestra (1988; LWP). The UV fluxes were integrated over the band $1450-1700 \AA$, with uncertainties evaluated as in Falomo et al. (1993). Notice that in 3 years the source has brightened by $\sim 40 \%$ (Fig. 5).

Strong emission lines are apparent in both the optical $(\mathrm{H} \gamma$, $\mathrm{H} \beta$, and [O III]) and UV ( $\mathrm{Ly} \alpha, \mathrm{Si}$ IV, C IV, C III]) spectra (see 

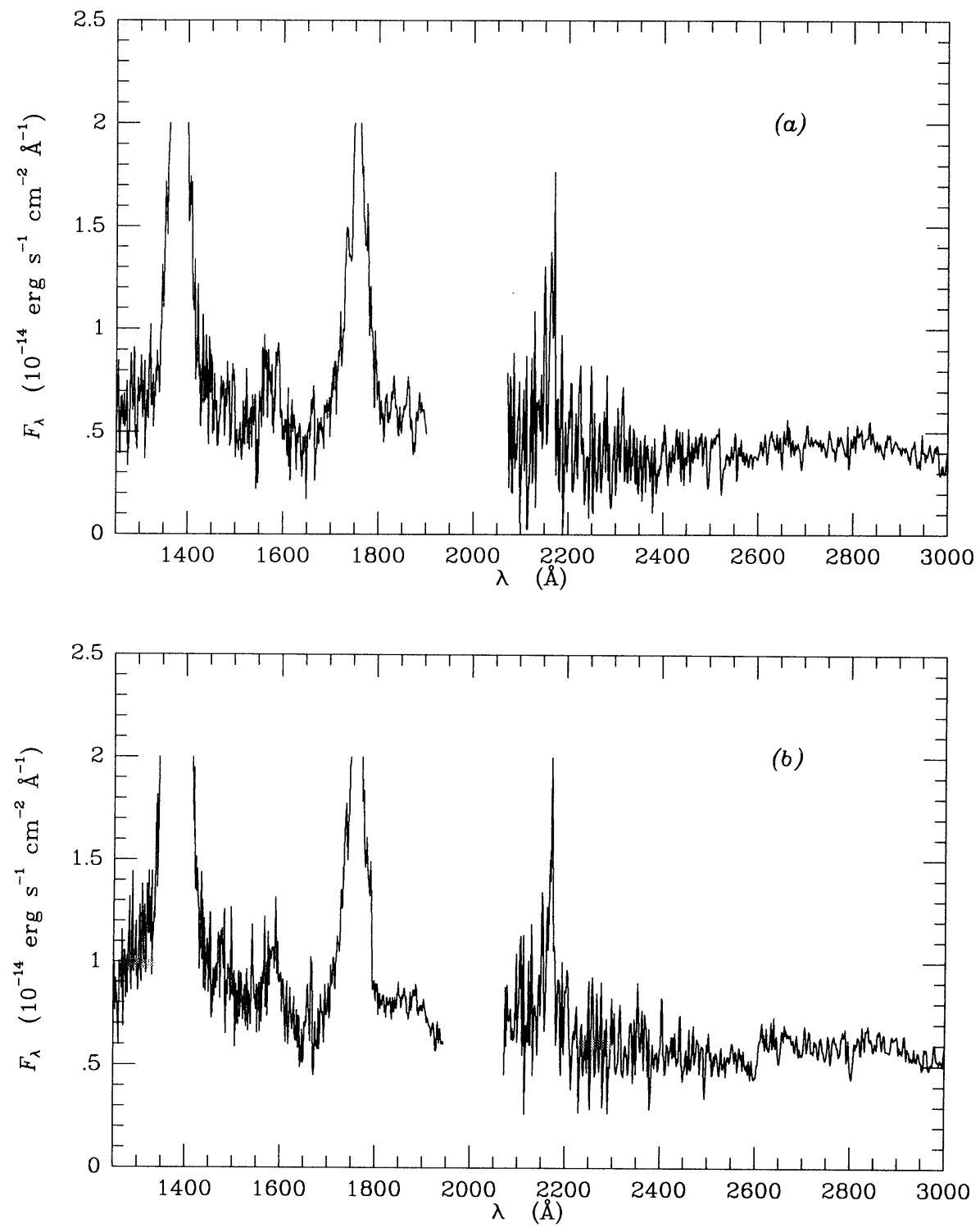

Fig. 5.-IUE spectra: (a) 1987 May 22-23 (SWP 31032 and LWP 10810); (b) 1990 October 14 (SWP 39831 and LWP 19011). The Ly $\alpha$ and C IV emission lines are truncated.

Table 3 and Figs 4 and 5). The intensities and equivalent widths are evaluated with errors of $10 \%-20 \%$, mainly due to the uncertainty in the estimate of the underlying continuum. The intensity ratios are typical for quasars (see, e.g., Netzer 1990).

\subsection{Photometry}

Optical photometry was performed in various broadband filters at the Lick Observatory, Capilla Peak Observatory (New Mexico), and at the Heidelberg Landessternwarte (Tables 4 and 5).

Twenty-three nights of direct imaging were done on the Lick Observatory $1 \mathrm{~m}$ Nickel telescope using a $500 \times 500$ pixel CCD. Eighteen of these nights were either photometric or very nearly so. The field of view was 4.5 on a side and the plate scale was $\sim 0$. 6 pixel $^{-1}$. High-precision $(2 \%-3 \%)$ relative photometry was obtained by using nine comparison stars to adjust all frames to a common flux level. Observations were made with Johnson B, Gunn $g$, and Kron-Cousins $I$ filters. An additional 10 nights of direct imaging were obtained on the Lick 3

TABLE 3

UV AND OPTICAL EMISSION LINES

\begin{tabular}{crr}
\hline \hline Ion & \multicolumn{1}{c}{$I^{\mathrm{a}}$} & EW $^{\mathrm{b}}$ \\
\hline Ly $\alpha \lambda 1216 \ldots \ldots \ldots$ & 220 & 210 \\
Si IV $\lambda 1403 \ldots \ldots \ldots$ & 19 & 26 \\
C IV $\lambda 1549 \ldots \ldots \ldots$ & 86 & 100 \\
C III $\lambda 1909 \ldots \ldots \ldots$ & 13 & 17 \\
H $\gamma \lambda 4340 \ldots \ldots \ldots$ & 9 & 42 \\
H $\beta \quad \lambda 4861 \ldots \ldots \ldots$ & 17 & 90 \\
{$[$ O III $] \lambda 5007 \ldots \ldots$} & 5 & 29 \\
\hline
\end{tabular}

a Observed line intensity in $10^{-14}$ ergs $\mathrm{s}^{-1} \mathrm{~cm}^{-2}$.

${ }^{b}$ Observed line equivalent width in angstroms. 
TABLE 4

OPTICAL PHOTOMETRY

\begin{tabular}{|c|c|c|c|c|c|}
\hline Date & $\boldsymbol{B}$ & $g$ & $V$ & $R$ & $I$ \\
\hline \multicolumn{6}{|c|}{ Lick Observatory } \\
\hline $\begin{array}{l}1990 \text { Aug } 01 \ldots \ldots \\
1990 \text { Aug } 21 \ldots \ldots \\
1990 \text { Aug } 27 \ldots \ldots \\
1990 \text { Aug } 29 \ldots \ldots \\
1990 \text { Aug } 31 \ldots \ldots \\
1990 \text { Sep } 11 \ldots \ldots \\
1990 \text { Sep } 12 \ldots \ldots \\
1990 \text { Sep } 13 \ldots \ldots \\
1990 \text { Sep } 15 \ldots \ldots \\
1990 \text { Sep } 21 \ldots \ldots \\
1990 \text { Sep } 22 \ldots \ldots \\
1990 \text { Sep } 29 \ldots \ldots \\
1990 \text { Oct } 12 \ldots \ldots \\
1990 \text { Oct } 16 \ldots \ldots \\
1990 \text { Oct } 17 \ldots \ldots \\
1990 \text { Oct } 29 \ldots \ldots \\
1990 \text { Nov } 11 \ldots \ldots \\
1991 \text { Feb } 20 \ldots \ldots \\
1991 \text { Feb } 21 \ldots \ldots \\
1991 \text { Sep } 03 \ldots \ldots \\
1991 \text { Sep } 05 \ldots \ldots\end{array}$ & $\begin{array}{c}\ldots \\
\ldots \\
\ldots \\
\ldots \\
15.662 \pm 0.054 \\
15.655 \pm 0.007 \\
15.661 \pm 0.010 \\
15.668 \pm 0.019 \\
15.644 \pm 0.005 \\
15.703 \pm 0.011 \\
15.679 \pm 0.006 \\
\ldots \\
15.741 \pm 0.014 \\
\ldots \\
15.706 \pm 0.014 \\
15.755 \pm 0.054 \\
\ldots \\
\ldots \\
15.627 \pm 0.011 \\
15.849 \pm 0.029 \\
15.846 \pm 0.010\end{array}$ & $\begin{array}{r}15.688 \pm 0.010 \\
15.655 \pm 0.018 \\
\ldots \\
15.652 \pm 0.013 \\
15.616 \pm 0.054 \\
15.653 \pm 0.012 \\
15.653 \pm 0.015 \\
15.661 \pm 0.011 \\
15.651 \pm 0.054 \\
15.681 \pm 0.020 \\
15.690 \pm 0.007 \\
15.677 \pm 0.028 \\
15.768 \pm 0.054 \\
15.715 \pm 0.021 \\
15.692 \pm 0.017 \\
15.705 \pm 0.054 \\
15.700 \pm 0.009 \\
15.690 \pm 0.038 \\
\ldots \\
15.823 \pm 0.006 \\
15.828 \pm 0.021\end{array}$ & 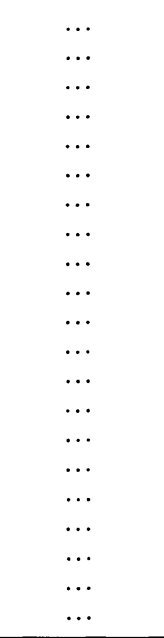 & 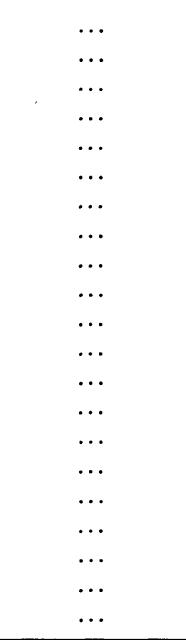 & $\begin{array}{c}15.385 \pm 0.017 \\
\ldots \\
15.359 \pm 0.012 \\
15.351 \pm 0.006 \\
15.349 \pm 0.054 \\
15.371 \pm 0.008 \\
15.335 \pm 0.009 \\
15.398 \pm 0.015 \\
15.367 \pm 0.037 \\
15.384 \pm 0.011 \\
15.380 \pm 0.026 \\
15.354 \pm 0.015 \\
15.430 \pm 0.054 \\
15.431 \pm 0.048 \\
15.395 \pm 0.026 \\
15.432 \pm 0.054 \\
\ldots \\
\ldots \\
\ldots \\
\ldots\end{array}$ \\
\hline \multicolumn{6}{|c|}{ Capilla Peak Observatory (New Mexico) } \\
\hline $\begin{array}{l}1990 \text { Sep } 21 \ldots \ldots \\
1990 \text { Sep } 28 \ldots \ldots \\
1990 \text { Oct } 13 \ldots \ldots\end{array}$ & $\begin{array}{c}\cdots \\
15.99 \pm 0.05 \\
15.85 \pm 0.05\end{array}$ & $\begin{array}{l}\cdots \\
\cdots \\
\cdots\end{array}$ & $\begin{array}{l}15.44 \pm 0.04 \\
15.62 \pm 0.04 \\
15.54 \pm 0.04\end{array}$ & $\begin{array}{c}\cdots \\
15.20 \pm 0.06 \\
15.27 \pm 0.05\end{array}$ & $\begin{array}{l}14.45 \pm 0.08 \\
14.49 \pm 0.09 \\
14.52 \pm 0.08\end{array}$ \\
\hline \multicolumn{6}{|c|}{ Landessternwarte Heidelberg } \\
\hline $\begin{array}{l}1990 \text { Sep } 13 \ldots \ldots \\
1990 \text { Sep } 13 \ldots \ldots \\
1990 \text { Sep } 14 \ldots \ldots \\
1990 \text { Sep } 16 \ldots \ldots \\
1990 \text { Sep } 16 \ldots \ldots \\
1990 \text { Sep } 28 \ldots \ldots \\
1990 \text { Oct } 03 \ldots \ldots \\
1990 \text { Oct } 08 \ldots \ldots \\
1990 \text { Oct } 09 \ldots \ldots \\
1990 \text { Oct } 10 \ldots \ldots \\
1990 \text { Oct } 22 \ldots \ldots \\
1990 \text { Oct } 23 \ldots \ldots \\
1990 \text { Dec } 05 \ldots \ldots \\
1990 \text { Dec } 06 \ldots \ldots \\
1990 \text { Dec } 07 \ldots \ldots\end{array}$ & $\begin{array}{l}\ldots \\
\cdots \\
\cdots \\
\cdots \\
\cdots \\
\cdots \\
\cdots \\
\cdots \\
\cdots \\
\cdots \\
\cdots \\
\cdots \\
\cdots \\
\cdots \\
\cdots \\
\end{array}$ & $\begin{array}{l}\cdots \\
\cdots \\
\cdots \\
\cdots \\
\cdots \\
\cdots \\
\cdots \\
\cdots \\
\cdots \\
\cdots \\
\cdots \\
\cdots \\
\cdots \\
\cdots \\
\cdots \\
\end{array}$ & $\begin{array}{l}\ldots \\
\ldots \\
\ldots \\
\ldots \\
\ldots \\
\ldots \\
\ldots \\
\ldots \\
\ldots \\
\ldots \\
\ldots \\
\ldots \\
\ldots \\
\ldots \\
\ldots \\
\end{array}$ & $\begin{array}{l}15.139 \pm 0.018 \\
15.173 \pm 0.018 \\
15.187 \pm 0.018 \\
15.192 \pm 0.018 \\
15.205 \pm 0.019 \\
15.200 \pm 0.019 \\
15.218 \pm 0.019 \\
15.226 \pm 0.019 \\
15.205 \pm 0.019 \\
15.202 \pm 0.019 \\
15.261 \pm 0.020 \\
15.262 \pm 0.020 \\
15.247 \pm 0.019 \\
15.222 \pm 0.019 \\
15.193 \pm 0.018 \\
\end{array}$ & $\begin{array}{l}\cdots \\
\cdots \\
\cdots \\
\cdots \\
\cdots \\
\cdots \\
\cdots \\
\cdots \\
\cdots \\
\cdots \\
\cdots \\
\cdots \\
\cdots \\
\cdots \\
\cdots \\
\end{array}$ \\
\hline
\end{tabular}

$\mathrm{m}$ Shane telescope using the same filters. Eight of these nights were photometric. The $3 \mathrm{~m}$ has a smaller field of view so there were only two-three comparison stars in common with the $1 \mathrm{~m}$ field. The $1 \mathrm{~m}$ and $3 \mathrm{~m}$ frames were combined into one data set for input into the computer code which performed the relative photometry calculations. Any frame which had only one good comparison star was arbitrarily given an error bar of $5 \%$, about twice the average observed error. The observations were referenced to spectrophotometric standard stars in Oke (1974), Oke \& Gunn (1983), and Stone (1977). The resulting light curves are shown in Figure 1.

The Capilla Peak Observatory uses a $1.05 \mathrm{~m}$ telescope equipped with a CCD. Broadband photometry was obtained with the $B, V, R$, and $I$ Johnson-Kron-Cousins filters. Absolute calibration is based on the Hayes \& Latham (1975) calibration of Vega using the effective wavelengths for the filters given by Landolt (1983).

Photometry at $\sim 6200 \AA$ was also performed between 1990 September 13 and December 7 with the $70 \mathrm{~cm}$ telescope at the
Landessternwarte Heidelberg equipped with a CCD (GEC, $576 \times 386$ pixel, 0 ".85 pixel $^{-1}$ ) with a Johnson $R$ filter (Table 4 , bottom section). The integration time for each exposure was $\sim 1200$ s. Due to the observing conditions, no absolute calibration could be performed. The reduction included bias subtraction and flat fielding via "sky flats." The fluxes of the source and of several comparison stars were measured using the Daophot package. By computing the normalized ratios of the target fluxes versus the comparison stars the light curve was derived (Fig. 1e). The error bars correspond to $1 \sigma$ uncertainties. The fluxes were then calibrated according to the Capilla Peak $R$ photometry, on the basis of two simultaneous observations (September 28) and the calibrated magnitudes are given in Table 4.

The photometric data in the different bands and the spectrophotometry at the corresponding effective wavelengths are generally consistent to within $\sim 10 \%-15 \%$.

Table 5 shows a comparison of the mean photometry taken at three different telescopes. Agreement is good $(\lesssim 10 \%)$ in all 
TABLE 5

X-Ray, UV, and Optical Properties

\begin{tabular}{|c|c|c|c|c|c|}
\hline Band & $\begin{array}{c}\lambda^{\mathbf{a}} \\
(\AA)\end{array}$ & Instrument & $\begin{array}{c}\langle F\rangle \\
(\mathrm{mJy})\end{array}$ & $\sigma_{F}^{\mathrm{b}}$ & $\chi^{2}$ \\
\hline$X$. & 12.4 & $R O S A T+P S P C$ & $0.85 \times 10^{-3}$ & 0.199 & 1.33 \\
\hline UV ....... & 1600 & $I U E+\mathrm{SWP}$ & 0.75 & 0.100 & 11.7 \\
\hline$B^{\prime} \ldots \ldots$ & 4250 & $\mathrm{CCD}^{\mathrm{c}}+$ Johnson & 1.90 & 0.065 & 47.6 \\
\hline$B \ldots \ldots$ & 4400 & $C C D^{d}+J K C$ & 1.94 & & \\
\hline$g^{\prime} \ldots \ldots \ldots$ & 4900 & $C C D^{c}+G u n n$ & 1.92 & 0.050 & 38.0 \\
\hline & 5500 & $\mathrm{CCD}^{\mathrm{d}}+\mathrm{JKC}$ & 2.23 & & \\
\hline$R^{\prime} \ldots \ldots$ & 6200 & $\mathrm{CCD}^{\mathrm{e}}+$ Johnson & $1.00^{f}$ & 0.030 & 4.02 \\
\hline$R \ldots \ldots$ & 6800 & $\mathrm{CCD}^{\mathrm{d}}+\mathrm{JKC}$ & 2.37 & & \\
\hline$I^{\prime} \ldots \ldots \ldots$ & 8000 & $C C D^{c}+K C$ & 2.56 & 0.029 & 4.70 \\
\hline$I \ldots \ldots \ldots$ & 8250 & $\mathrm{CCD}^{\mathrm{d}}+\mathrm{JKC}$ & 3.87 & $\ldots$ & $\ldots$ \\
\hline
\end{tabular}

${ }^{\text {a }}$ Central wavelength.

b Variability index calculated (only for $N>3$ ) as in Edelson 1992

$$
\sigma_{F}=\frac{1}{\langle F\rangle}\left[\frac{1}{N-1} \sum_{i=1}^{N}\left(F_{i}-\langle F\rangle\right)^{2}\right]^{1 / 2}
$$

c On the Lick Observatory $1 \mathrm{~m}$ and $3 \mathrm{~m}$ telescopes.

d On the Capilla Peak Observatory $1.05 \mathrm{~m}$ telescope.

- On the Landessternwarte Heidelberg $70 \mathrm{~cm}$ telescope.

${ }^{f}$ Relative normalization.

cases except the $I$ band, which shows a $40 \%$ discrepancy. This discrepancy has not been resolved and we thus adopt a larger error for the $I$ points $( \pm 20 \%)$.

The optical-UV light curves indicate some dimming of the source in the first part of the observation, most prominently in the $B$ band. A brightening at MJD $=8300$ is apparent in $B$ and UV light curves. Comparable $\mathrm{X}$-ray variability may be masked by the large uncertainties.

\section{DISCUSSION}

The light curves at the different frequencies show some variability of the source during our monitoring. In Table 5 we give the intrinsic variability parameter for the various bands. The variability increases regularly with increasing frequency. There is a suggestion of a correlation between the various bands, but unfortunately the UV and optical points are too scarce and unevenly spaced to look for lags between the various light curves. Moreover, the uncertainties on the X-ray flux are rather large, which prevents a reliable determination of lags (see, e.g., Edelson et al. 1995, for a discussion on these points).

Our observations allow us to construct the simultaneous composite continuum spectrum from the $I$ band to the X-rays, which is given in Figure 6. Both $I$ fluxes obtained by us are reported. UV and optical continuum fluxes have been corrected for the extinction with $A_{V}=0.3$, based on the Galactic column density $N_{\mathrm{H}}=5.0 \times 10^{20}$ (Elvis et al. 1989), a gas-todust ratio $N_{\mathrm{H}} / E_{B-V}=5.2 \times 10^{21} \mathrm{~cm}^{-2} \mathrm{mag}^{-1}$ (Shull \& Van Steenberg 1985) and $R=3.1$ (Rieke \& Lebofsky 1985) for the ratio of total to selective extinction. The dereddening follows Seaton (1979). The UV-optical data correspond to 1990 October 14 and 15 , respectively, while for the X-rays we report the power-law fit of the averaged ROSAT spectrum. For comparison, the Einstein spectrum is also reported.

The median radio-quiet quasar spectral energy distribution proposed by Elvis et al. (1994) has been superposed on the present data, after suitable rescaling to the redshift of the object, and it reproduces satisfactorily the overall spectrum (see Fig. 6). In the $I$ band there is a spectral rise, which is characteristic of the class. McDowell, Elvis, \& Wilkes (1992),

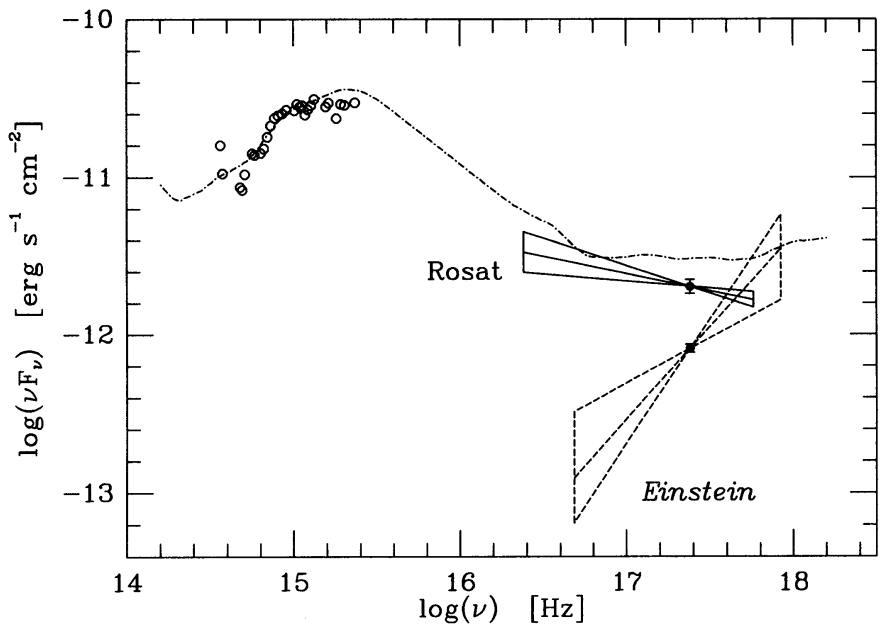

FIG. 6.-Overall spectral energy distribution. The open dots represent the I-band fluxes (Capilla Peak, 1990 October 13; and Lick Observatory, October 16), the optical (MMT, 1990 October 15) and the UV (IUE with SWP and LWP, October 14) data. The solid lines correspond to the best fit and $1 \sigma$ confidence range of the average ROSAT spectrum. The dashed lines are the fit and $1 \sigma$ confidence range of the 1979 Einstein observation. The dotted-dashed curve represents the median radio-quiet quasar spectral energy distribution as from Elvis et al. (1994).

on the basis of IRAS observations, noted that the source is relatively infrared weak above $\sim 10 \mu \mathrm{m}$. We have no new infrared data to confirm this weakness. The $1200-4300 \AA$ range is well fitted by a power law of energy index $\alpha_{v} \simeq 0.9\left(F_{v} \propto v^{-\alpha_{v}}\right)$, which is typical for radio-quiet quasars (see, for PG quasars, Pian \& Treves 1993).

In Figure 6 it can be seen that an extrapolation of the ROSAT X-ray spectrum underestimates the UV flux by a factor of $\sim 5$. The interpolation from $1500 \AA$ to $1 \mathrm{keV}$ yields a slope $\alpha_{v}=1.5$. The fact that the UV data lie above the extrapolation of the X-rays suggests the presence of a soft X-ray excess (Walter \& Fink 1993), which is not unusual for this class of objects. However, evidence for a soft component within the $\mathrm{X}$-ray data is weak, since the best-fit column density is close to the Galactic one, and the single power-law fit is acceptable $\left(\chi^{2} \simeq 0.9\right)$. On the other hand, the soft component may ease the problem of matching the UV to the X-rays. Taking a temperature of $21 \mathrm{eV}$ (see Table 1) corresponding to the blackbody + power law best fitted to the ROSAT spectrum, one can account for $10 \%$ of the $1200 \AA$ flux. The linear dimension of the black body emitting region is $10^{12}-10^{13} \mathrm{~cm}$, a value somewhat lower than those considered for thermal emission in quasars (Malkan \& Sargent 1982). If the blackbody temperature were lower, one could account for a larger fraction of the UV, and at the same time increase the size of the region responsible for thermal emission. However, the fraction of the total blackbody flux which enters in the ROSAT range (0.1-2.4 $\mathrm{keV}$ ), which is $50 \%$ for $T=21 \mathrm{eV}$, would become substantially smaller and the hypothesis of the thermal component would appear even more ad hoc. The same considerations prevent us from searching for a fit with a more complex thermal component as, e.g., an accretion disk spectrum.

The ROSAT X-ray slope is rather typical of radio-quiet quasars. The sample of 21 radio-quiet quasars observed with Einstein $(0.2-3.5 \mathrm{keV})$ gives in fact $\langle\Gamma\rangle=2.0 \pm 0.1$ (Shastri et al. 1993), while a sample of 53 radio-quiet quasars observed with $R O S A T$ yielded $\langle\Gamma\rangle=2.54 \pm 0.04$ (Schartel, Walter, \& 
Fink 1994), indicating that within this sample Kaz 102 is among those with harder spectra.

The most important result of our observations is that the ROSAT spectrum is substantially softer than that obtained from the Einstein data. It seems hard to ascribe such a large difference to the different energy ranges of the two instruments which do not overlap completely. Some systematic differences between indices measured with the two instruments are indeed reported by various authors. However, these are around $\Delta \Gamma \simeq 0.6-0.7$ (Fiore et al. 1994; Schartel et al. 1994). Brunner et al. (1994) find for a sample of radio-selected quasars that the Einstein IPC spectral slopes are consistent with the ROSAT ones at $90 \%$ confidence. The difference $\Delta \Gamma=1.1$ found by us (see $\S 2.2$ ) with $5 \sigma$ confidence appears to be significant. Therefore we are led to consider that the variation is real. The spectrum remains steep for the entire duration of the ROSAT observation and its slope is consistent with the average value of radio-quiet quasars, thus probably a somewhat unusual state was detected by Einstein, which leads the object at the extreme of the hardness distribution of quasars and makes it an inter- esting target for observation at higher energies. The fact that in the low state the spectrum is very flat and in the higher state a thermal component may show up, indicate that the energy distribution is rather complicated and that the power-law fits, even if formally acceptable are most probably inadequate.

The flux variations in the X-rays, between the Einstein and ROSAT observations, and the UV do not appear exceptional for a quasar if compared with the archives (Cutri et al. 1985; Maccacaro, Garilli, \& Mereghetti 1987; O’Brien, Gondhalekar, \& Wilson 1988; Kinney et al. 1991). Based on the spectral variability observed with EXOSAT $(0.1-8 \mathrm{keV})$ in a sample of bright active galactic nuclei (mostly Seyfert galaxies; Grandi et al. 1992), the variability is not unprecedented.

We thank J. McDowell and M. Elvis for critical reading of the manuscript. S. J. W. and J. H. acknowledge partial support by the DFG through SFB 328. The ROSAT mission is supported by the Ministerium für Forschung und Technologie (BMFT), Germany, and by the Max-Planck-Gesellschaft.
Bohlin, R., Harris, A. W., Holm, A. V., \& Gry, C. 1990, ApJS, 73, 413

Brinkmann, W., \& Trümper, J. 1992, MPE Rep., No. 235

Brunner, H., Lamer, G., Worrall, D. M., \& Staubert, R. 1994, A\&A, 287, 436

Cassatella, A., Lloyd, C., \& Gonzalez-Riestra, R. 1988, IUE ESA Newsletter, 31,13 Cutri, R. M., Wisniewski, W. Z., Rieke, G. H., \& Lebofsky, M. J. 1985, ApJ,

Edelson, R. A. 1992, ApJ, 401, 516

Edelson, R. A., et al. 1995, ApJ, 438, 120

Elvis, M., et al. 1994, ApJS, 95, 1

Elvis, M., Lockman, F. J., \& Wilkes, B. J. 1989, AJ, 97, 777

Falomo, R., Treves, A., Chiappetti, L., Maraschi, L., Pian, E., \& Tanzi, E. G. 1993, ApJ, 402, 532

Fiore, F., Elvis, M., McDowell, J. C., Siemiginowska, A., \& Wilkes, B. J. 1994 ApJ, 431, 515

Grandi, P., Tagliaferri, G., Giommi, P., Barr, P., \& Palumbo, G. 1992, ApJS 82,93

Hayes, D. S. \& Latham, D. W. 1975, ApJ, 197, 593

Kinney, A. L., Bohlin, R. C., Blades, J. C., \& York, D. G. 1991, ApJS, 75, 645

Landolt, A. 1983, AJ, 88, 439

Maccacaro, T., Garilli, B., \& Mereghetti, S. 1987, AJ, 93, 1484

Malkan, M. A., \& Sargent, W. L. W. 1982, ApJ, 254, 22

McDowell, J. C., Elvis, M., \& Wilkes, B. J. 1992, in AIP Conf. Proc. 254 Testing the AGN Paradigm, ed. S. Holt, S. G. Neff, \& C. M. Urry (New York: AIP), 532

\section{REFERENCES}

Morrison, R., \& McCammon, D. 1983, ApJ, 270, 119

Netzer, H. 1990, in Proc Saas-Fee Advanced Course 20, Active Galactic Nuclei, ed. T. J.-L. Courvoisier \& M. Mayor (Berlin: Springer), 62 O'Brien, P. T., Gondhalekar, P. M., \& Wilson, R. 1988, MNRAS, 233, 845 Oke, J. B. 1974, ApJS, 27, 21

Oke, J. B., \& Gunn, J. E. 1983, ApJ, 266, 713

Pian, E., \& Treves, A. 1993, ApJ, 416, 130

Rieke, G. H., \& Lebofsky, M. J. 1985, ApJ, 288, 618

Schaeidt, S. 1994, Ph.D. thesis, Ludwig-Maximilian Universität, München

Schartel, N., Walter, R., \& Fink, H. H. 1994, A\&A, submitted

Seaton, M. J. 1979, MNRAS, 187, 73p

Shastri, P., Wilkes, B. J., Elvis, M., \& McDowell, J. C. 1993, ApJ, 410, 29

Shull, J. M., \& Van Steenberg, M. E. 1985, ApJ, 294, 599

Snowden, S. L., McCammon, D., Burrows, D. N., \& Mendenhall, J. A. 1994, ApJ, 424, 714

Stone, R. P. S. 1977, ApJ, 218, 767

Treves, A., et al. 1992, MPE Rep., No. 235, p. 175

Urry, C. M., \& Reichert, G. 1988, IUE NASA Newsletter, 34, 96

Walter, R., \& Fink, H. H. 1993, A\&A, 274, 105

Wilkes, B. J., \& Elvis, M. 1987, ApJ, 323, 243 